\title{
Sarcopenia and Osteoporotic Fractures
}

$$
\text { Itamar Levinger }{ }^{1,2} ; \text { Steven } \mathrm{Phu}^{1,3} \text {; Gustavo Duque }{ }^{1,3}
$$

${ }^{1}$ Australian Institute for Musculoskeletal Science (AIMSS), The University of Melbourne and Western Health, Melbourne, Australia.

${ }^{2}$ Clinical Exercise Science Research Program, Institute of Sport, Exercise and Active Living (ISEAL), College of Sport and Exercise Science, Victoria University, Melbourne, Australia.

${ }^{3}$ Department of Medicine, Melbourne Medical School - Western Campus, The University of Melbourne, St. Albans, Australia.

Abbreviated title: Sarcopenia and Fractures

Correspondence to: Prof. Gustavo Duque, MD, Ph.D., FRACP

Australian Institute for Musculoskeletal Science (AIMSS)

Melbourne Medical School - Western Campus

The University of Melbourne

176 Furlong Road, St. Albans, VIC, 3021, Australia

Tel: +61 383958121

Email: gustavo.duque@unimelb.edu.au 


\begin{abstract}
Low bone mass is strongly associated with increased fracture risk. However, the importance of low muscle mass and strength - known as sarcopenia - as a risk factor for osteoporotic fractures remains overlooked and sometimes controversial. Bone and muscle are closely interconnected not only anatomically, but also physically, chemically and metabolically. Indeed, a significant proportion of individuals with sarcopenia also suffer from osteopenia/osteoporosis suggesting a link between the two tissues. This subgroup of osteosarcopenic individuals are at higher risk of falls and fractures. Therefore, we suggest that lean mass and muscle strength/function assessments should be an integral part in any fracture prevention protocol. A combination of lean mass quantification by dual-energy X-ray absorptiometry scan and assessment of muscle function by gait velocity could not only confirm the diagnosis of sarcopenia but also optimize any fracture prevention interventions. In absence of specific therapies for sarcopenia, simple interventions such as resistance (weight bearing) training, protein supplements and appropriate levels of vitamin D have a dual effect on bone and muscle, and could have a significant effect on reducing falls and fractures in this high risk population.
\end{abstract}

Keywords: Osteoporosis; sarcopenia; osteosarcopenia; fractures, falls, elderly. 


\section{INTRODUCTION}

The annual loss of muscle mass with aging is between 1 to $2 \%$ per year after the age of 50 years $[1,2]$. In the case of bone, a similar decline occurs from the third decade of life, being more severe during the menopause years in women. When the age-related decline in bone mass is more than $2 \%$ per year, the bone becomes brittle and prone to fracture thus fulfilling the diagnostic criteria for osteoporosis [3]. In the muscle, age-related changes could be aggravated by other factors (i.e. disuse, hypogonadism, malnutrition, etc.) that affect not only muscle mass but also muscle function and strength, which fulfill the diagnostic criteria for sarcopenia $[1,2,4]$. Bone and muscle are closely interconnected not only physically but also chemically and metabolically. This connection is important because muscle mass closely correlates with bone mass and any concurrent decrease in both tissues is associated with higher risk of falls and fractures [5]. The mechanisms that explain the synchronic loss of bone and muscle mass are multiple: 1) low muscle mass is associated with abnormal glucose metabolism and changes in muscle-related proteins, known as myokines, which have a direct effect on bone metabolism [6];

2) decreased muscle strength and physical performance is associated with low mechanical loading thus directly affecting bone mass [3]; 3) sarcopenic individuals are at higher risk of falls, which also predispose to fractures.

From the diagnostic perspective, sarcopenia and osteoporosis could be simultaneously assessed by dual energy X-ray absorptiometry (DXA) looking at muscle - using lean mass as a surrogate and bone mineral density (BMD) respectively. In the case of sarcopenia, muscle (lean) mass assessment should be complemented with other clinical variables such as gait velocity and grip strength. Although there is a consensus that DXA is an accurate method to quantify muscle mass, there is still controversy regarding the clinical diagnostic criteria for sarcopenia [7-9]. In addition, since sarcopenia and osteoporosis are closely linked, the term osteosarcopenia has been proposed to describe a subset of frailer individuals suffering from both sarcopenia and osteopenia/osteoporosis, and at higher risk of institutionalization, falls, and fractures [10, 11]. 
Based on the particular clinical characteristics and poor outcomes observed in individuals with osteosarcopenia, sarcopenia should be included as a critical indicator of higher fracture risk. Therefore, limiting fracture risk assessment to BMD and bone-related risk factors without taking into consideration the presence of sarcopenia could overlook a significant proportion of individuals that, despite having normal BMD or no bone-related risk factors, are still at a high fracture risk.

From the therapeutic perspective, the presence of sarcopenia would also have a significant role in the response to fracture prevention strategies. Although current drugs for osteoporosis have no effect on muscle mass, and considering that there are no current pharmacological approaches to sarcopenia, interventions that are known to improve sarcopenia, such as exercise and protein supplements, could also have an effect on bone mass and should be encouraged in osteosarcopenic patients. In this review, we will highlight the clinical relevance of sarcopenia in terms of fracture risk assessment. Initially, we will summarize new knowledge on bone and muscle communication. We will then review the clinical characteristics and recently described syndromes that involve sarcopenia as their key clinical feature. Finally, we will suggest a clinical pathway to diagnose osteosarcopenia, which we expect would facilitate clinical decisions in this particular high-risk population.

\section{THE MUSCLE AND BONE INTERFACE}

Until recently, bones and muscles were perceived to function independently despite the fact that both are part of the musculoskeletal system. In recent years, however, great interest was placed on the relationship and the cross-talk between bone and muscle as sarcopenia and osteoporosis have become more prevalent due to the aging population.

There are many factors affecting both muscle and bone, which may indicate a close relationship between the two tissues (Figure 1). Recent review papers describe in detail the potential factors affecting both bone and muscle $[6,12]$. As such, in this review we will briefly discuss the effects 
of aging, mechanical loading exercise, and unloading (bed rest/disuse) and the interaction between osteocalcin, a marker of bone formation and skeletal muscle.

\section{Aging}

Both sarcopenia and a reduction in aerobic capacity are part of the normal aging process [13-15]. Between the second and the seventh decades of life, there is a $30 \%$ reduction in muscle strength, a $25-40 \%$ reduction in muscle mass and muscle cross-sectional area, and also $8-10 \%$ reduction in maximal aerobic capacity each decade [16-18]. In addition, between the third and seventh decades, there is around $30 \%$ reduction in bone mass [19]. These changes in both muscle and bone occur in healthy individuals but are accelerated in those with chronic disease or in the presence of specific risk factors. It is not yet clear why aging has such a profound effect on these two tissues, even in individuals who maintain an active lifestyle. However, it appears that increased low level of systemic and local inflammatory markers, including mitogen activated protein kinases (MAPK), nuclear factor kappa-B (NF- $\mathrm{KB})$, c-Jun N-terminal kinases (JNK) and the signal transducer and activator of transcription (STAT), tumor necrosis factor (TNF $\alpha$ ), interleukin (IL)-6, IL8, IL-1 $\beta$ and monocyte chemotactic protein-1 (MCP1), are involved in the development of sarcopenia, osteoporosis and other chronic diseases in older adults [20-22]. The increase in inflammatory markers may shift the delicate balance between protein synthesis and degradation in muscle and bone formation and resorption in bone in the favor of degradation (muscle) / resorption (bone) leading to sarcopenia and bone loss [21].

\section{Mechanical loading and unloading}

The musculoskeletal system is a mechanosensing system. It can sense a change in individual load (body mass) or other forms of loading/stress (such as exercise) or unloading (micro-gravity, bed rest and disuse). Bone and muscle have the ability to adapt to mechanical loading by modifying their mass and strength [23]. There is a similarity between the alteration of skeletal muscle and 
bone due to aging that is caused by deconditioning and disuse [14, 24, 25]. For instance, it has been reported that 4-14.4 months of micro-gravity environment (Mir space station) resulted in a decrease (range 0.35 to $1.56 \%$ per month) in BMD as well as a reduction in leg lean mass ( $1 \%$ per month) [26]. This decline was observed despite an "extensive exercise countermeasure program". Similarly, a significant reduction in muscle mass and strength and BMD was reported following bed rest $[27,28]$.

As was described previously by Isaacson and Brotto [23], mechanical stimulus is essential for both muscle and bone health. In contrast to the decline in muscle and bone mass in response to inactivity, mechanical loading (especially resistance and weight bearing exercises), increases muscle mass and strength, and has the potential to improve BMD [29]. It is possible that muscle contraction in response to a mechanical loading, as occurs during resistance exercise, increases strain on bone, which, in turn, stimulates bone formation. Indeed, there is a correlation between muscle and bone strength [30]. However, it is important to note that not all studies that reported an increase in muscle mass and muscle strength following resistance training also reported an increase in BMD [29]. In fact, there are conflicting data in regards to the ability of exercise training to modify bone mass [31]. For instance, McCartney et al. [32] reported that 42 weeks of progressive resistance training resulted in 5.5\% increase in the knee extensors cross-sectional areas and $65 \%$ increase in muscle strength with no change in BMD. These data may suggest that the interaction between muscle and bone is more complicated and perhaps involves other factors than mechanical loading/stress alone. It may also suggest that muscle and bone require different exercise intensities and duration to elicit structural changes in both tissues. Indeed, muscle hypertrophy can be visible within several weeks of resistance training whereas changes in BMD may be present in exercise intervention that are greater than 6-12 months. 


\section{Osteocalcin and skeletal muscle}

Both muscle and bone are now recognized as endocrine organs with both playing a role in glycemic control [33-36]. There is increasing evidence that osteocalcin (OC), a marker of bone formation [37], in its undercarboxylated form (ucOC) stimulates $\beta$-cell proliferation and insulin secretion and ucOC-deficient mice are obese and glucose intolerant [34]. In humans, ucOC, and OC, are also correlated with insulin sensitivity, fasting glucose, fat mass and muscle strength [3841]. There is also increased evidence the ucOC act directly on skeletal muscle. Recently, we [42] and others [43] demonstrated that ucOC improves insulin-stimulated glucose uptake in $\mathrm{C} 2 \mathrm{C} 12$ myotubes. Furthermore, ucOC treatment improves muscle (EDL) insulin stimulated glucose uptake following an ex vivo muscle contraction [42]. The ucOC treatment promoted insulininduced Akt phosphorylation. In humans, it has been reported that high-intensity exercise increased ucOC as well as p-AKT and p-AS160 [35]. Both p-AKT and p-AS160 are downstream proteins in the PI3-K pathway regulating GLUT-4 translocation and as such are important for muscle glucose uptake capacity $[44,45]$.

It is important to note that the area of bone-muscle interaction is relatively new, and many of the mechanisms involved in the "cross-talk" between bone and muscle are not yet well understood. It is possible that the "cross-talk" between bone and muscle is bidirectional and factors that are released by muscle, especially during muscle contraction, can act on bone and vice versa (Figure 2) [6].

\section{SARCOPENIA, OSTEOSARCOPENIA AND SARCOPENIC OBESITY: DEFINITION AND CLINICAL DIFFERENCES}

\section{Sarcopenia}

As previously described, sarcopenia is a disease in which there is a gradual, generalized loss of muscle mass associated with the aging process and aggravated by the presence of other risk factors. In the clinical setting, sarcopenia has been defined as having an appendicular skeletal 
mass (ASM) divided by height in meters squared which was two standard deviations below that of a young healthy adult as assessed by DXA [46]. There is a consensus that this loss of muscle mass is typically combined with decline in muscle strength and/or physical function [4]. The assessment of muscular strength via handgrip dynamometer is commonly performed and has been described as an easily conducted measure, with a correlation between upper and lower body strength, highlighting the generalized loss of muscle in sarcopenia [2]. Decline in physical performance and function is another defining component of sarcopenia, and can be assessed with a simple measure of gait velocity [9].

\section{Osteosarcopenia}

As previously described, older adults who present with both osteopenia/osteoporosis and sarcopenia are termed as being osteosarcopenic, or sarco-osteopenic/sarco-osteoporotic [47-48]. In addition to the previously mentioned criteria for sarcopenia, individuals with osteosarcopenia will also present with low BMD (osteopenic or osteoporotic) as measured by DXA [47]. These individuals are at higher risk of falls and fractures than sarcopenic individuals [11] and have particular nutritional and physical phenotypes [11, 49].

\section{Sarcopenic Obesity}

Sarcopenic obesity refers to the combination of excess body weight (obesity) and sarcopenia [50]. The development of obesity can be associated with a variety of factors including a lack of physical activity, poor diet and age related hormonal and metabolic changes [51-53]. Therefore, additionally to the loss of muscle mass, those diagnosed with sarcopenic obesity will also present with excess weight with waist circumference as a measure of the obesity level $[51,52]$. Recently, the term osteosarcopenic obesity was introduced to describe those overweight individuals also suffering from osteosarcopenia $[50,54]$. Although the specific differences between osteosarcopenic obese and non-obese remain unknown, specific abnormalities in vitamin D levels 
have been reported in this particular sub-population, which may increase their risk of falls and fractures [55].

\section{CLINICAL OUTCOMES ASSOCIATED WITH SARCOPENIC SYNDROMES}

Sarcopenia is prevalent in older adults. It is estimated that more than $50 \%$ of the people over the age of 80 years suffer from sarcopenia [56]. Sarcopenia has major clinical, functional and psychological consequences [56] and in combination with the prevalence of the condition it places vast personal and financial cost on individuals and the community.

Additionally to increased risk of falls and fractures, other clinical and functional consequences of sarcopenia include: inability to sustain muscular power and endurance and a reduction in the capacity to perform activities of daily living [57]. Low muscle mass and strength may also lead to postural and mobility problems and increased fall risk [58]. Ultimately, it can lead to a reduced quality of life and premature mortality [56]. In addition, muscle is a major site for glucose disposal, as such, a reduction in muscle mass may have some other clinical consequences including increased risk of insulin resistance and type 2 diabetes [59]. As such, the ability to maintain and increase muscle mass and strength, particularly via weight bearing (resistance) exercises, are crucial to minimizing the effects of sarcopenia on physical performance and fall risk [60].

\section{HOW TO PREDICT FRACTURES IN SARCOPENIC PATIENTS?}

Although the evidence linking sarcopenia and osteoporotic fractures is controversial [61], in this review we have provided a rationale to include sarcopenia within any fracture risk assessment protocol, which is supported by recent literature. A recent review by Oliveira and Raz [61] concluded that there is strong evidence on the association between sarcopenia, osteoporosis, and hip fracture, with most authors considering that sarcopenia is a predictor of fracture risk in the elderly, and provides incremental predictive value if integrated with BMD and other risk factors. 
However, the question remains on how to integrate sarcopenia assessment within a fracture risk assessment protocol. Although there are very few studies looking at this question, most of the existing data suggest that DXA is an accurate method to quantify muscle (lean) mass [62]. Since many DXA machines include body composition analysis, and considering the clinical benefits of identifying sarcopenia in older persons, this additional investigation could be useful as a routine test when performing DXA analyses allowing the diagnosis of both sarcopenia and osteopenia/osteoporosis with one imaging modality.

Current consensus statements on sarcopenia state that the clinical diagnosis requires both a low muscle (lean) mass as well as decreased muscle function and/or strength [4,9]. Once a low muscle (lean) mass is documented by DXA, it should be correlated with at least one clinical indicator of muscle function and/or strength. In clinical practice, gait velocity is a simple method to assess muscle function and identify sarcopenia. A recent study performed in a population of older fallers [49] found that the subgroup of osteosarcopenic individuals (individuals fulfilling the clinical and DXA criteria for sarcopenia and osteopenia/osteoporosis) had both slow gait velocity and lower BMD, thus suggesting that gait velocity is not only a useful diagnostic method for sarcopenia but also a strong predictor of poor outcomes (including low BMD and fractures) in this high risk population. In terms of other function/strength parameters to diagnose sarcopenia, knee extension and grip strength positively associate with BMD [62] but require a higher level of expertise in order to be accurately measured.

In conclusion, although there is limited evidence on sarcopenia as a predictor of osteoporotic fractures, it is well known that sarcopenia and osteoporosis are closely linked and that osteosarcopenic individuals are at higher risk of falls and fractures. Therefore, sarcopenia should be included as an important risk factor for osteoporosis and fractures that is easy to diagnose and that can be considered as an additional therapeutic target when developing fracture prevention strategies. 


\section{CONCLUSION}

Muscle and bone are closely linked from the mechanic to the metabolic perspective. Therefore, any fracture prevention approach should also include assessment of muscle mass, strength and function. In absence of specific medications for sarcopenia, there are other interventions that have demonstrated to be effective. These interventions include resistance training, appropriate vitamin D levels, and protein supplements [5]. In conclusion, when assessing fracture risk, the clinician should always consider the possibility that sarcopenia is also present. Simple interventions could have a direct effect on muscle mass thus reducing the risk of falls and fractures in this particular high-risk population.

\section{ACKNOWLEDGEMENTS}

A/Prof Levinger was supported by Future Leader Fellowship (ID: 100040) from the National Heart Foundation of Australia.

\section{CONFLICT OF INTEREST}

No conflict of interest to disclose

\section{COMPLIANCE WITH ETHICAL STANDARDS}

Human studies: All procedures performed in studies involving human participants were in accordance with the ethical standards of the institutional and/or national research committee and with the 1964 Helsinki declaration and its later amendments or comparable ethical standards. Informed consent was obtained from all individual participants included in the study. Animal studies: All procedures performed in studies involving animals were in accordance with the ethical standards of the institution or practice at which the studies were conducted. 


\section{REFERENCES}

[1] Hughes VA, Frontera WR, Roubenoff R, Evans WJ, Singh MA. Longitudinal changes in body composition in older men and women: role of body weight change and physical activity. Am J Clin Nutr. 2002;76:473-81.

[2] Lauretani F, Russo CR, Bandinelli S, Bartali B, Cavazzini C, Di Iorio A, Corsi AM, Rantanen T, Guralnik JM, Ferrucci L. Age-associated changes in skeletal muscles and their effect on mobility: an operational diagnosis of sarcopenia. J Appl Physiol. 2003;95:1851-60.

[3] Bevier WC, Wiswell RA, Pyka G, Kozak KC, Newhall KM, Marcus R. Relationship of body composition, muscle strength, and aerobic capacity to bone mineral density in older men and women. J Bone Miner Res. 1989;4:421-32.

[4] Cruz-Jentoft AJ, Baeyens JP, Bauer JM, Boirie Y, Cederholm T, Landi F, Martin FC, Michel JP, Rolland Y, Schneider SM, Topinková E, Vandewoude M, Zamboni M; European Working Group on Sarcopenia in Older People. Sarcopenia: European consensus on definition and diagnosis: Report of the European Working Group on Sarcopenia in Older People. Age Ageing. 2010;39:412-23.

[5] Cederholm T, Cruz-Jentoft AJ, Maggi S. Sarcopenia and fragility fractures. Eur J Phys Rehabil Med. 2013;49:111-7.

[6] Kawao N, Kaji H. Interactions between muscle tissues and bone metabolism. J Cell Biochem. 2015;116:687-95.

[7] Cederholm T, Morley JE. Sarcopenia: the new definitions. Curr Opin Clin Nutr Metab Care. 2015;18:1-4.

[8] McLean RR, Kiel DP. Developing consensus criteria for sarcopenia: an update. J Bone Miner Res. 2015;30:588-92.

[9] Fragala MS, Dam TT, Barber V, Judge JO, Studenski SA, Cawthon PM, McLean RR, Harris TB, Ferrucci L, Guralnik JM, Kiel DP, Kritchevsky SB, Shardell MD, Vassileva MT, Kenny AM. Strength and function response to clinical interventions of older women categorized by 
weakness and low lean mass using classifications from the Foundation for the National Institute of Health sarcopenia project. J Gerontol A Biol Sci Med Sci. 2015;70:202-9.

[10] Drey M, Sieber CC, Bertsch T, Bauer JM, Schmidmaier R; FiAT intervention group. Osteosarcopenia is more than sarcopenia and osteopenia alone. Aging Clin Exp Res. 2015 Nov 12.

[11] Huo YR, Suriyaarachchi P, Gomez F, Curcio CL, Boersma D, Muir SW, Montero-Odasso M, Gunawardene P, Demontiero O, Duque G. Phenotype of osteosarcopenia in older individuals with a history of falling. J Am Med Dir Assoc. 2015;16:290-5.

[12] Isaacson J, Brotto M. Physiology of Mechanotransduction: How Do Muscle and Bone "Talk" to One Another? Clin Rev Bone Miner Metab. 2014;12:77-85.

[13] Landi F, Calvani R, Cesari M, Tosato M, Martone AM, Bernabei R, Onder G, Marzetti E. Sarcopenia as the Biological Substrate of Physical Frailty. Clin Geriatr Med. 2015;31:36774.

[14] Hagerman FC, Walsh SJ, Staron RS, Hikida RS, Gilders RM, Murray TF, Toma K, Ragg KE. Effects of high-intensity resistance training on untrained older men. I. Strength, cardiovascular, and metabolic responses. Biol Sci. 2000;55A:B336-46.

[15] Mishra SK, Misra V. Muscle sarcopenia: an overview. Acta Myol. 2003;22:43-7.

[16] Fletcher GF, Balady G, Amsterdam EA, Chaitman B, Eckel R, Fleg JL, Froelicher VF, Leon AS, Pina IL, Rodney R, Simons-Morton DG, Williams MA, Bazzarre T. Exercise standards for testing and training: a statement for healthcare professionals from the American Heart Association. Circulation. 2001;104:1694-1740.

[17] Oxenham H, Sharpe N. Cardiovascular aging and heart failure. Eur J Heart fail. $2003 ; 5: 427-34$

[18] Proctor DN, Joyner MJ. Skeletal muscle mass and the reduction of $\mathrm{VO}_{2}$ max in trained older subjects. J Appl Physiol. 1997;82:1411-15. 
[19] Frost HM. On our age-related bone loss: insights from a new paradigm. J Bone Miner Res. 1997;12:1539-46.

[20] Caldow MK, Cameron-Smith D, Levinger P, McKenna MJ, Levinger I. Inflammatory markers in skeletal muscle of older adults. Eur J Appl Physiol. 2013;113:509-17.

[21] Levinger I, Levinger P, Trenerry MK, Feller JA, Bartlett JR, Bergman N, McKenna MJ, Cameron-Smith D. Increased inflammatory cytokine expression in the vastus lateralis of patients with knee osteoarthritis. Arthritis Rheum. 2011;63:1343-48.

[22] Pawelec G, Goldeck D, Derhovanessian E. Inflammation, ageing and chronic disease. Curr Opin Immunol. 2014;29:23-28.

[23] Goodman CA, Hornberger TA, Robling AG. Bone and skeletal muscle: Key players in mechanotransduction and potential overlapping mechanisms. Bone. 2015;80:24-36.

[24] Bloomfield AS. Changes in musculoskeletal structure and function with prolonged bed rest. Med Sci Sports Exerc. 1997;29:197-206.

[25] Convertino VA, Bloomfield AS, Greenleaf JE. An overview of the issues: physical effects of bed rest and restricted physical activity. Med Sci Sports Exerc. 1997;29:187-190.

[26] LeBlanc A, Schneider V, Shackelford L, West S, Oganov V, Bakulin A, Voronin L. Bone mineral and lean tissue loss after long duration space flight. J Musculoskelet Neuronal Interact. 2000;1:157-160.

[27] Leblanc AD, Schneider VS, Evans HJ, Engelbretson DA, Krebs JM. Bone mineral loss and recovery after 17 weeks of bed rest. J Bone Miner Res. 1990;5:843-50.

[28] LeBlanc AD, Schneider VS, Evans HJ, Pientok C, Rowe R, Spector E. Regional changes in muscle mass following 17 weeks of bed rest. J Appl Physiol. 1992;73:2172-78.

[29] Layne JE, Nelson ME. The effects of progressive resistance training on bone density: a review. Med Sci Sports Exerc. 31: 25-30, 1999. 
[30] Bevier WC, Wiswell RA, Pyka G, Kozak KC, Newhall KM, Marcus R. Relationship of body composition, muscle strength, and aerobic capacity to bone mineral density in older men and women. J Bone Miner Res. 1989;4:421-32.

[31] Wallace BA, Cumming RG. Systematic review of randomized trials of the effect of exercise on bone mass in pre- and postmenopausal women. Calcif Tissue Int. 2000;67:10-18.

[32] McCartney N, Hicks AL, Martin J, Webber CE. Long-term resistance training in the elderly: effects on dynamic strength, exercise capacity, muscle, and bone. J Gerontol A Biol Sci Med Sci. 1995;50: B97-B104.

[33] Confavreux CB, Levine RL, Karsenty G. A paradigm of integrative physiology, the crosstalk between bone and energy metabolisms. Mol Cell Endocrinol. 2009;310:21-29.

[34] Lee NK, Sowa H, Hinoi E, Ferron M, Ahn JD, Confavreux C, Dacquin R, Mee PJ, McKee MD, Jung DY, Zhang Z, Kim JK, Mauvais-Jarvis F, Ducy P, Karsenty G. Endocrine regulation of energy metabolism by the skeleton. Cell. 2007;130: 456-69.

[35] Levinger I, Jerums G, Stepto NK, Parker L, Serpiello FR, McConell GK, Anderson M, Hare DL, Byrnes E, Ebeling PR, Seeman E. The effect of acute exercise on undercarboxylated osteocalcin and insulin sensitivity in obese men. J Bone Miner Res. 2014;29:2571-6.

[36] Pedersen BK, Febbraio MA. Muscle as an endocrine organ: focus on muscle-derived interleukin-6. Physiol Rev. 2008;88:1379-406.

[37] Booth SL, Centi A, Smith SR, Gundberg C. The role of osteocalcin in human glucose metabolism: marker or mediator? Nat Rev Endocrinol. 2013;9: 43-55.

[38] Fernandez-Real JM, Izquierdo M, Ortega F, Gorostiaga E, Gomez-Ambrosi J, MorenoNavarrete JM, Fruhbeck G, Martinez C, Idoate F, Salvador J, Forga L, Ricart W, Ibanez J. The relationship of serum osteocalcin concentration to insulin secretion, sensitivity, and disposal with hypocaloric diet and resistance training. J Clin Endocrinol Metab. 94: 237-245, 2009. 
[39] Kanazawa I, Yamaguchi T, Yamamoto M, Yamauchi M, Kurioka S, Yano S, Sugimoto T. Serum osteocalcin level is associated with glucose metabolism and atherosclerosis parameters in type 2 diabetes mellitus. J Clin Endocrinol Metab. 94: 45-49, 2009.

[40] Kanazawa I, Yamaguchi T, Yamauchi M, Yamamoto M, Kurioka S, Yano S, Sugimoto T. Serum undercarboxylated osteocalcin was inversely associated with plasma glucose level and fat mass in type 2 diabetes mellitus. Osteoporos Int. 2011;22:187-94.

[41] Levinger I, Scott D, Nicholson GC, Stuart AL, Duque G, McCorquodale T, Herrmann M, Ebeling PR, Sanders KM. Undercarboxylated osteocalcin, muscle strength and indices of bone health in older women. Bone. 2014;64:8-12.

[42] Levinger I, Lin X, Zhang X, Brennan-Speranza TC, Volpato B, Hayes A, Jerums G, Seeman E, McConell G. The effects of muscle contraction and recombinant osteocalcin on insulin sensitivity ex vivo. Osteopor Int. 2015.

[43] Tsuka S, Aonuma F, Higashi S, Ohsumi T, Nagano K, Mizokami A, KawakuboYasukochi T, Masaki C, Hosokawa R, Hirata M, Takeuchi H. Promotion of insulin-induced glucose uptake in C2C12 myotubes by osteocalcin. Biochem Biophys Res Commun. 2015;459:437-42.

[44] Cartee GD, Wojtaszewski JF. Role of Akt substrate of $160 \mathrm{kDa}$ in insulin-stimulated and contraction-stimulated glucose transport. Appl Physiol Nutr Metab. 32: 557-566, 2007.

[45] Krook A, Wallberg-Henriksson H, Zierath JR. Sending the signal: molecular mechanisms regulating glucose uptake. Med Sci Sports Exerc. 2004;36:1212-17.

[46] Baumgartner RN, Koehler KM, Gallagher D, Romero L, Heymsfield SB, Ross RR, Garry PJ, Lindeman RD. Epidemiology of sarcopenia among the elderly in New Mexico. Am J Epidemiol. 1998;147:755-63.

[47] Kull M, Kallikorm R, Lember M. Impact of a new sarco-osteopenia definition on healthrelated quality of life in a population-based cohort in Northern Europe. J Clin Densitom. $2012 ; 15: 32-38$ 
[48] Binkley N, Buehring B. Beyond FRAX: it's time to consider "sarco-osteopenia". J Clin Densitom. 2009;12:413-6.

[49] Huo YR, Suriyaarachchi P, Gomez F, Curcio CL, Boersma D, Gunawardene P, Demontiero O, Duque G. Comprehensive nutritional status in sarco-osteoporotic older fallers. J Nutr Health Aging. 2015;19:474-80.

[50] Ormsbee MJ, Prado CM, Ilich JZ, Purcell S, Siervo M, Folsom A, Panton L. Osteosarcopenic obesity: the role of bone, muscle and fat on health. J Cachexia Sarcopenia Muscle. 2014;5:183-92

[51] Stenholm S, Harris T, Rantanen T, et al. Sarcopenic obesity - definition, etiology and consequences. Curr Opin Clin Nutr Metab Care. 2008;11:693-700

[52] Ryu M, Jo J, Lee Y, Chung YS, Kim KM, Baek WC.Association of physical activity with sarcopenia and sarcopenic obesity in community-dwelling older adults: the Fourth Korea National Health and Nutrition Examination Survey. Age and Ageing 2013;42:734-40

[53] Atkins JL, Whincup PH, Morris RW, Lennon LT, Papacosta O, Wannamethee SG. Sarcopenic obesity and risk of cardiovascular disease and mortality: a population-based cohort study of older men. J Am Geriatr Soc 2014;62:253-60

[54] Ilich JZ, Kelly OJ, Inglis JE, Panton LB, Duque G, Ormsbee MJ. Interrelationship among muscle, fat, and bone: connecting the dots on cellular, hormonal, and whole body levels. Ageing Res Rev. 2014;15:51-60.

[55] Hita-Contreras F, Martínez-Amat A, Cruz-Díaz D, Pérez-López FR.

Osteosarcopenic obesity and fall prevention strategies. Maturitas. 2015;80:126-32.

[56] Cruz-Jentoft AJ, Landi F, Topinkova E, Michel JP. Understanding sarcopenia as a geriatric syndrome. Curr Opin Clin Nutr Metab Care.13: 1-7, 2010.

[57] Evans WJ, Campbell WW. Sarcopenia and age-related changes in body composition and functional capacity. J Nutr. 1993;123:465-8. 
[58] Landi F, Liperoti R, Russo A, Giovannini S, Tosato M, Capoluongo E, Bernabei R, Onder G. Sarcopenia as a risk factor for falls in elderly individuals: results from the ilSIRENTE study. Clin Nutr. 201;31:652-8.

[59] Srikanthan P, Karlamangla AS. Relative muscle mass is inversely associated with insulin resistance and prediabetes. Findings from the third National Health and Nutrition Examination Survey. J Clin Endocrinol Metab. 96: 2898-2903, 2011.

[60] LaStayo PC, Ewy GA, Pierotti DD, Johns RK, Lindstedt S. The positive effects of negative work: increased muscle strength and decreased fall risk in a frail elderly population. J Gerontol A Biol Sci Med Sci. 58: M419-424,

[61] Oliveira A, Vaz C. The role of sarcopenia in the risk of osteoporotic hip fracture. Clin Rheumatol. 2015;34:1673-80.

[62] Bijlsma AY, Meskers MC, Molendijk M, Westendorp RG, Sipilä S, Stenroth L, Sillanpää E, McPhee JS, Jones DA, Narici M, Gapeyeva H, Pääsuke M, Seppet E, Voit T, Barnouin Y, Hogrel JY, Butler-Browne G, Maier AB. Diagnostic measures for sarcopenia and bone mineral density. Osteoporos Int. 2013;24:2681-91. 


\section{FIGURE LEGENDS}

Figure 1. Factors affecting the interactions between muscle and bone. GH/IGF-I, growth hormone/insulin-like growth factor-I. Adapted from Kawao et al [6]

Figure 2. Humoral factors linking muscle to bone. FGF2, fibroblast growth factor 2; FAM5C, family with sequence similarity 5, member, C; IL, interleukin; MMP-2, matrix metalloproteinase2; MGF, mechano growth factor; VEGF, vascular endothelial growth factor; HGF, hepatocyte growth factor. Adapted from Kawao et al [6] 


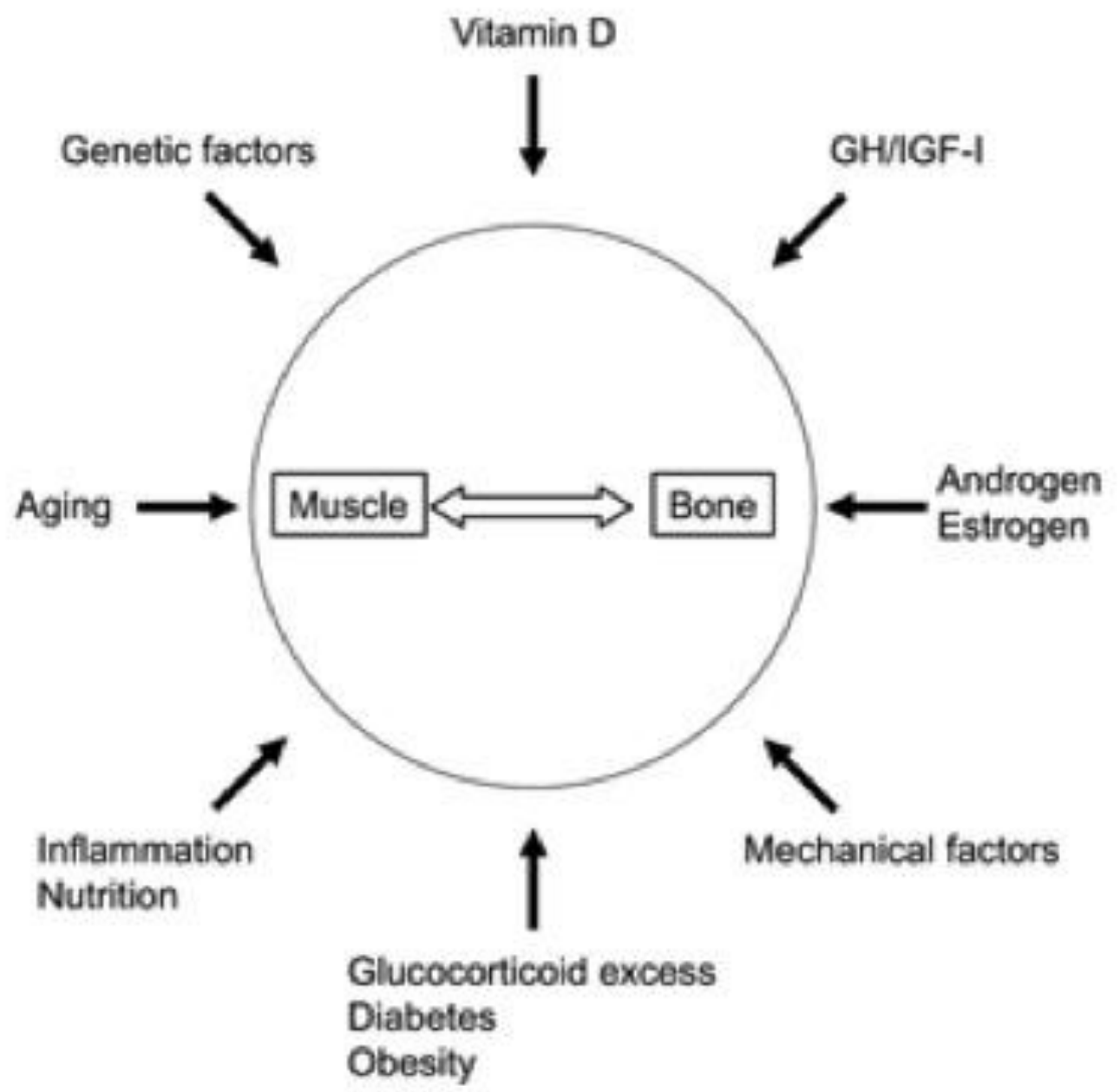


IGF-I, Myostatin, Osteoglycin,

FAM5C, Irisin, Osteonectin, FGF2,

IL-6, IL-7, IL-15, MMP-2

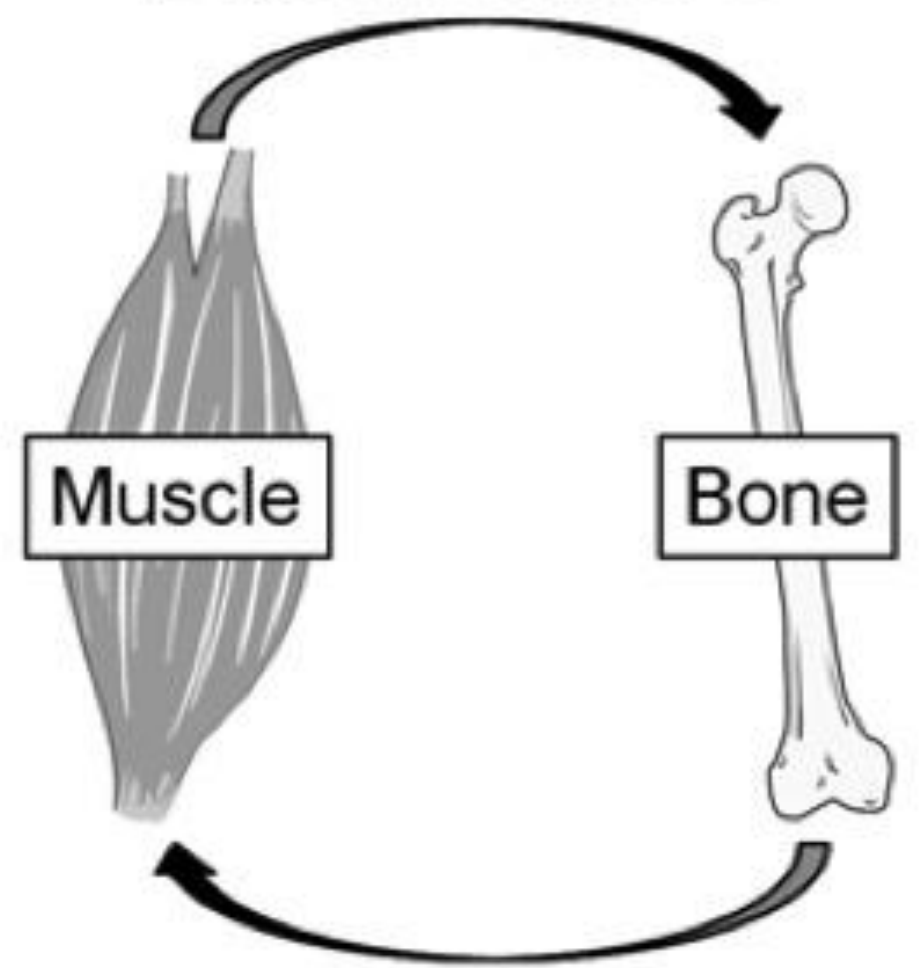

IGF-I, Sclerostin, Osteocalcin, MGF, VEGF, HGF 